\title{
Atribuição de falsas crenças no desenvolvimento de linguagem de crianças com síndrome de Down
}

\author{
Attribution of false beliefs in the language development of \\ children with Down syndrome
}

\author{
Tatiana Pires da Silva ${ }^{1}$, Amanda Fernandes da Silva ${ }^{2}$, Ana Carina Tamanaha ${ }^{3}$, Jacy Perissinoto ${ }^{4}$
}

\begin{abstract}
RESUMO
Objetivo: Avaliar atribuição de falsa crença em indivíduos com Síndrome de Down. Métodos: Onze crianças usuárias de comunicação verbal, com síndrome de Down, retardo mental de grau leve a grave, de ambos os sexos, na faixa etária entre quatro e oito anos e atendidas em instituição compuseram o Grupo Down (GD). Além disso, 85 crianças sem alterações do desenvolvimento, na faixa etária entre quatro e seis anos, matriculadas em EMEI, constituíram o Grupo Controle (GC). Foram utilizados o Teste de Vocabulário por Imagem Peabody (TVIP) para a comparação do nível de compreensão verbal dos grupos, e o "teste dos smarties" adaptado, para avaliar a atribuição de falsa crença. Resultados: Na análise do TVIP verificou-se diferença estatisticamente significante entre os grupos, sendo que o GD apresentou pontuação abaixo do terceiro desvio-padrão e, as crianças do GC, abaixo do primeiro desviopadrão. Em relação à análise da atribuição de falsa crença, o GC apresentou progressão de acertos em todas as questões conforme o aumento da faixa etária. O mesmo não foi observado para o GD, sendo que os melhores resultados foram os dos indivíduos com maior tempo de terapia fonoaudiológica na instituição. Não houve correlação entre o nível de vocabulário receptivo e a habilidade de falsa crença. Conclusão: Em todas as questões houve melhor desempenho do GC em comparação ao GD. Sendo assim, foi possível analisar a falsa crença em crianças com síndrome de Down.
\end{abstract}

Descritores: Síndrome de Down; Linguagem infantil; Desenvolvimento infantil; Processos mentais; Cognição

\section{INTRODUÇÃO}

Muitas são as teorias que estudam o surgimento da linguagem e grande parte delas correlaciona o seu desenvolvimento ao da cognição e à inserção social.

A interação social é crucial para a aquisição da linguagem. Neste sentido, a linguagem é um ato social que requer prática. Quando os pais imitam os sons dos bebês eles os ajudam a

Trabalho realizado no Laboratório de Investigações Fonoaudiológicas nos Transtornos Globais do Desenvolvimento (LIF-TGD), do Departamento de Fonoaudiologia da Universidade Federal de São Paulo - UNIFESP - São Paulo (SP), Brasil, como parte de projeto de iniciação científica financiado pelo Conselho Nacional de Desenvolvimento Científico e Tecnológico - CNPq.

(1) Fonoaudióloga do Laboratório de Investigação Fonoaudiológica nos Transtornos Globais do Desenvolvimento da Universidade Federal de São Paulo - UNIFESP - São Paulo (SP), Brasil.

(2) Professora substituta do Curso de Fonoaudiologia da Universidade Federal de Sergipe - UFS - Aracaju (SE), Brasil.

(3) Doutora, Professora convidada do Departamento de Fonoaudiologia da Universidade Federal de São Paulo - UNIFESP - São Paulo (SP), Brasil.

(4) Doutora, Professora Associada do Departamento de Fonoaudiologia da Universidade Federal de São Paulo - UNIFESP - São Paulo (SP), Brasil.

Endereço para correspondência: Tatiana Pires da Silva. R. Botucatu, 802, Vl. Clementino, São Paulo (SP), Brasil, CEP: 04023-062. E-mail: tatipires@msn.com

Recebido em: 8/3/2009; Aceito em: 8/9/2009 experimentar o aspecto social da fala com as mudanças de entonação e trocas de turnos, por exemplo.

Em contrapartida, estudos sobre a relação entre desenvolvimento cognitivo e o desempenho de linguagem, e suas implicações sociais e educacionais em condição adversa, como a encontrada em indivíduos com síndrome de Down, merecem observações e acompanhamento cuidadoso de profissionais especializados ${ }^{(1)}$.

A linguagem é a área na qual a criança com síndrome de Down apresenta maior atraso. Usualmente estas crianças compreendem bem mais as informações do que as produzem verbalmente. Apresentam dificuldades articulatórias que podem persistir até a vida adulta. Apesar disso, a maioria dos indivíduos faz uso funcional da linguagem e compreende as regras utilizadas nas conversações ${ }^{(1)}$.

O desenvolvimento da linguagem destes indivíduos passapelas mesmas fases que o desenvolvimento de linguagem típico, porém de maneira mais lenta e por vezes não completa. Alguns teóricos constataram que crianças com síndrome de Down atingem linguagem semelhante a de crianças com desenvolvimento normal, até cinco anos ${ }^{(1,2)}$.

Em relação às habilidades sociais, pesquisas com crianças com síndrome de Down revelaram a capacidade adequada em atribuir estados mentais, correlacionando este fato com sua 
habilidade social intacta, embora os prejuízos intelectuais sejam evidentes ${ }^{(3)}$.

Baron-Cohen empregou o modelo de meta-representação para correlacionar o desenvolvimento cognitivo à capacidade social humana. Este modelo teórico, denominado de Teoria da Mente, avalia a habilidade da criança em representar estados mentais e prever comportamentos de seus interlocutores ${ }^{(4)}$.

A meta-representação é requerida nos padrões sociais, sendo uma manifestação da relação entre o desenvolvimento cognitivo e de linguagem na criança. É necessária para o desenvolvimento de padrões simbólicos, como o faz-de-conta, o jogo simbólico, a criatividade e a originalidade. Sem ela, não é possível que haja predição do comportamento social ${ }^{(5,6)}$.

Aos 18 meses a criança típica já tem a compreensão de que as pessoas operam com metas e intenções. Aos quatro ou cinco anos de idade já desenvolveu uma Teoria da Mente bastante sofisticada, ou seja, é hábil em partilhar as percepções e os pensamentos com outras pessoas e, além disso, compreender falsas crenças. Também é capaz de atribuir estados mentais, tais como sentimentos e intenções, a si mesma e aos outros. Posteriormente, essas capacidades transformam-se numa habilidade essencial para a inserção social e cultural da criança ${ }^{(5,6)}$.

Teoria da Mente é a teoria psicológica que usamos para prever e explicar o comportamento do outro com base no seu funcionamento interno, seus sentimentos, intenções, desejos, atitudes, crenças, conhecimentos e pontos de vista e, desta maneira, predizer seu comportamento ou ação ${ }^{(5,6)}$.

Vários estudos têm investigado como as crianças desenvolvem a habilidade de compreender seus estados mentais e os de outras pessoas com as quais interagem, e de utilizarem esta informação para explicar e predizer o comportamento social. A partir dos cinco anos, elas são capazes de atribuir estados mentais a seus parceiros, no tocante à falsa crença $a^{(7)}$.

Desta forma, as crianças são capazes de se colocar na perspectiva do outro e distinguir crenças verdadeiras das falsas. Por exemplo, ao se considerar uma situação-problema na qual a criança tem que analisar a situação através da perspectiva do outro, é necessário que a mesma seja capaz de predizer corretamente a resposta alheia.

O objetivo deste estudo foi verificar se crianças com síndrome de Down, associada a algum grau de prejuízo cognitivo, são capazes de atribuir falsa crença, mesmo em idade mais avançada, quando comparados aos indivíduos cujo desenvolvimento é típico.

\section{MÉTODOS}

\section{Aspectos éticos}

Todos os pais e/ou responsáveis estavam cientes dos procedimentos do estudo e entregaram assinado o Termo de Consentimento Livre e Esclarecido, de acordo com as sugestões do Comitê de Ética em Pesquisa da instituição de origem (0600/06).

\section{Sujeitos}

A amostra deste estudo foi constituída por 11 crianças, na faixa etária entre quatro e oito anos, de ambos os gêneros, pertencentes a classes sócio-econômicas diversas (média de quatro salários mínimos por família), cujos pais apresentaram em média 8,8 anos de estudo formal, diagnosticadas com síndrome de Down, segundo comprovação laboratorial genética, tendo quatro delas associação com retardo mental leve, quatro com retardo mental moderado e três com retardo mental grave, segundo critérios diagnósticos do CID $10^{(8)}$, e todas atendidas em uma mesma instituição (GD).

No Grupo Controle (GC) foram avaliadas 85 crianças, sem evidências e/ou queixa de alterações do desenvolvimento motor, cognitivo e de linguagem, na faixa etária de quatro a seis anos, de ambos os sexos e pertencentes à classe sócioeconômica baixa (média de dois salários mínimos por família), cujos pais apresentavam, em média, 7,8 anos de estudo formal, e todas matriculadas numa mesma escola municipal de educação infantil.

Como critério de inclusão da amostra, considerou-se a presença de desempenho verbal no GD e a concordância da família para a pesquisa, em ambos os grupos.

Considerou-se critério de exclusão, evidência de comprometimentos associados, tais como alterações motoras, auditivas e/ou visuais, em ambos os grupos.

\section{Material}

A fim de caracterizarmos a habilidade de compreensão verbal de cada grupo do estudo e compará-los a partir de um mesmo referencial adotamos o Teste de Vocabulário por Imagem Peabody (TVIP) ${ }^{(9)}$. Este instrumento tem como objetivo avaliar o desenvolvimento lexical no domínio receptivoauditivo de indivíduos entre dois e 18 anos de idade por meio do reconhecimento de 125 figuras.

Para a verificação da atribuição de falsas crenças pela criança utilizamos uma variação da proposta original de Perner et al. ${ }^{(10)}$. Trata-se de uma situação-problema na qual é oferecida à criança uma caixa de lápis de cor em cujo interior é depositado apenas um clipe. A criança deve responder o que pensa ter dentro da caixa, sem tocá-la. Após responder a criança é incentivada a abrir a caixa e dizer o que há dentro dela. Em seguida, $o$ avaliador the propõe a seguinte situação-problema: "O que você acha que a sua professora irá responder quando eu perguntar a ela o que tem dentro da caixa, se ela apenas a tiver visto fechada?".

Oferecemos um clipe numa caixa de lápis de cor, ao contrário da proposta original que propunha um lápis dentro de uma caixa de bombons para evitarmos que o desenho do doce se tornasse um distrator.

Para garantirmos o reconhecimento da caixa de lápis por parte da criança, foi introduzida como primeira questão: "Isto é uma caixa do quê?".

Outra modificação apresentada foi a inclusão da questão 3: "Você sabe o que é isto?", a fim de garantirmos o reconhecimento do clipe como um objeto diferente do lápis.

Sendo assim, a sequência de questões ficou da seguinte maneira: 1) Isto é uma caixa do quê??; 2) O que você acha que tem dentro dessa caixa?; 3) Você sabe o que é isto? (após o avaliador abrir a caixa e mostrar o que tem dentro); 4) $O$ 
que você acha que a sua professora irá responder quando eu perguntar a ela o que tem dentro da caixa, se ela apenas a tiver visto fechada?

\section{Procedimento}

Cada criança foi avaliada individualmente num espaço cedido pela própria instituição. As avaliações foram gravadas e registradas em protocolo específico para este estudo.

Para as questões 1 e 2 foram aceitas respostas utilizando a descrição ou a função do lápis (ex: "é de pintar"). Para a questão 3 também foram aceitos sinônimo, descrição e/ou função do clipe, além de interjeições ou gestos que mostrassem à avaliadora que a criança havia reconhecido o objeto.

A questão 3 foi considerada de compreensão da situação e a questão 4 , de atribuição de falsa crença.

\section{Método estatístico}

Para o tratamento estatístico dos resultados obtidos em cada grupo na análise do desempenho comunicativo obtido na aplicação do TVIP, foi utilizado o teste da mediana e para análise das falsas crenças foi utilizado o teste Qui-quadrado, considerando-se 5\% de significância.

Para a análise de dados na avaliação das falsas crenças, houve agrupamento por faixa etária: $4 \mathrm{a} 6 \mathrm{~m}$ a $5 \mathrm{a} 5 \mathrm{~m}, 5 \mathrm{a} 6 \mathrm{~m}$ a $6 \mathrm{a} 5 \mathrm{~m}, 6 \mathrm{a} 6 \mathrm{~m}$ a $7 \mathrm{a} 5 \mathrm{~m}$ e $7 \mathrm{a} 6 \mathrm{~m}$ a $8 \mathrm{a} 5 \mathrm{~m}$ no GD e $4 \mathrm{a} 6 \mathrm{~m}$ a $5 \mathrm{a} 5 \mathrm{~m}$ e $5 \mathrm{a} 6 \mathrm{~m}$ a $6 \mathrm{a} 5 \mathrm{~m}$ no GC.

No GD consideramos ainda o tempo de intervenção terapêutica fonoaudiológica e o grau de retardo mental, obtidos a partir dos dados da avaliação psicológica e do registro de atendimentos realizados por profissionais da instituição.

\section{RESULTADOS}

A Figura 1 mostra a proporção da pontuação média convertida do TVIP, nos grupos GC e GD, evidenciando diferença nos resultados de cada grupo.

A Figura 2 mostra a proporção de acertos nas questões do teste de Falsa Crença no GC, distribuída por faixa etária, onde há diferença, principalmente na questão 4 , referente a atribuição da falsa crença.

A Figura 3 mostra a proporção de acertos nas questões do teste de Falsa Crença no GD, distribuída por faixa etária,

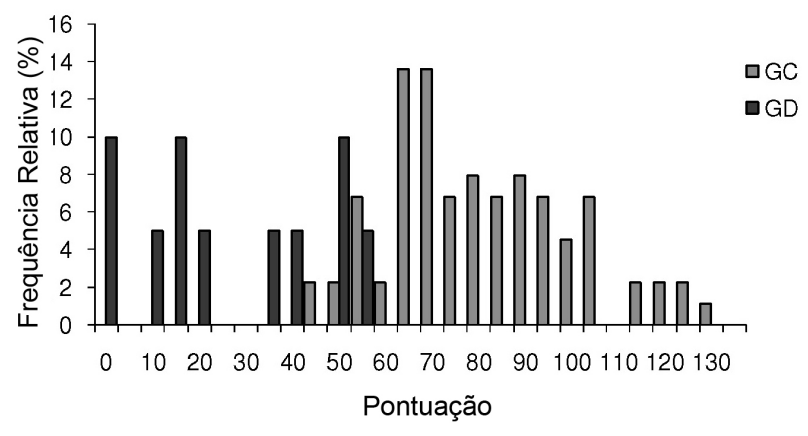

Figura 1. Pontuação do Teste de Vocabulário por Imagem Peabody (TVIP) no Grupo Down (GD) e Grupo Controle (GC)

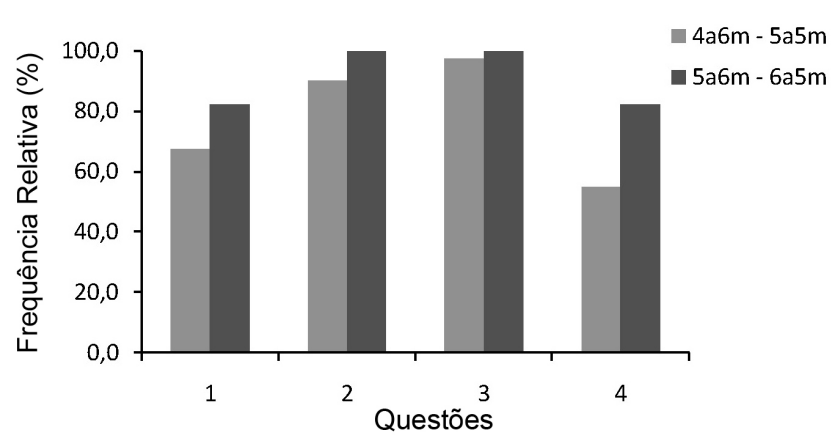

Figura 2. Proporção de acertos por faixa etária nas questões do teste de falsa crença para o Grupo Controle (GC)

indicando que apenas a partir dos cinco anos e seis meses as crianças passaram a apresentar a noção de falsa crença.

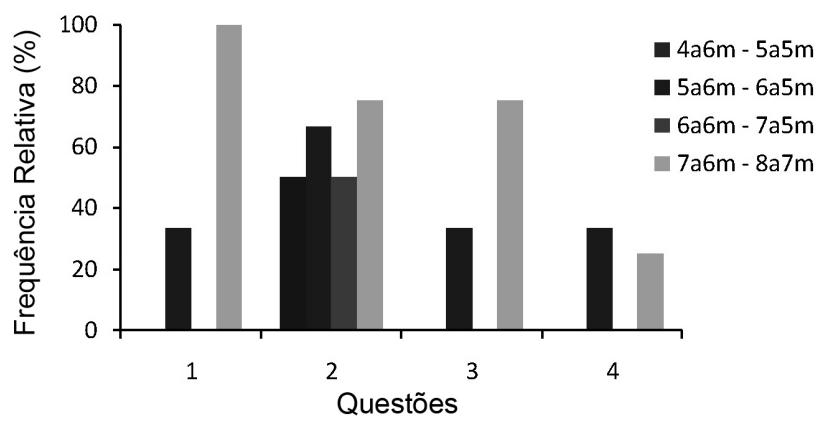

Figura 3. Proporção de acertos por faixa etária nas questões do teste de falsa crença para o Grupo Down (GD)

A Figura 4 mostra a distribuição dos indivíduos e seus acertos nas questões do teste de Falsa Crença no GD, distribuída por tempo de terapia fonoaudiológica, indicando que apenas as crianças com tempo de terapia maior de quatro anos e meio apresentaram esta habilidade.

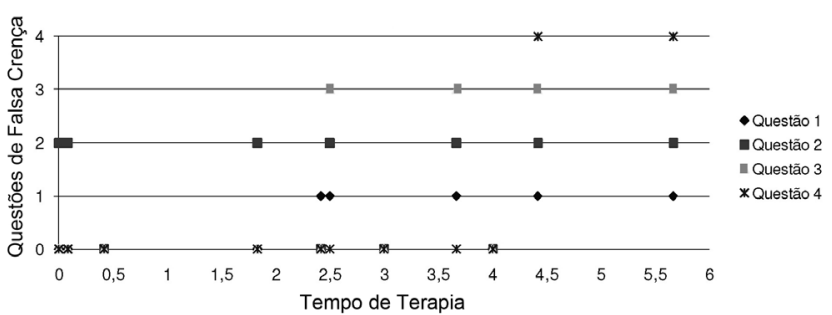

Figura 4. Disposição dos indivíduos e acertos nas questões do teste de falsa crença no Grupo Down (GD), por tempo de terapia fonoaudiológica, em anos. Nota: A figura apresenta as respostas de cada questão, onde o zero significa erro.

A Figura 5 mostra a proporção de acertos nas questões do teste de Falsa Crença no GD, distribuída por grau de retardo mental, sendo que apenas crianças com retardo mental leve foram capazes de atribuir falsas crenças.

Não foi encontrada relação estatisticamente significativa, em relação à idade, nem em relação ao gênero das crianças estudadas. 


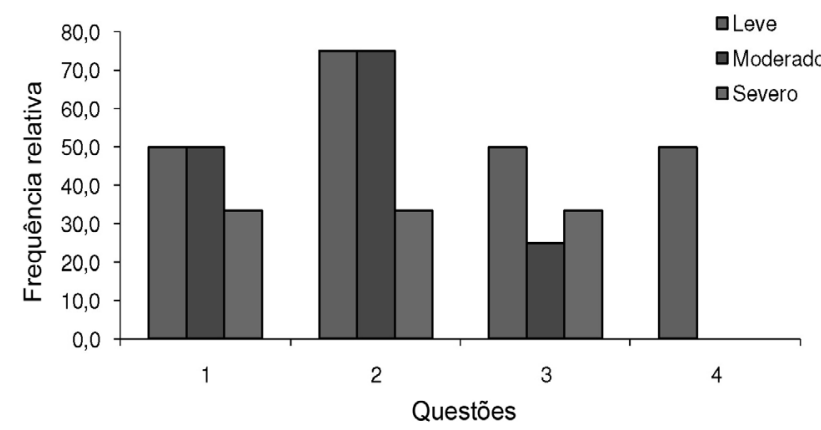

Figura 5. Proporção de acertos nas questões do teste de falsa crença por grau de retardo mental, no Grupo Down (GD)

\section{DISCUSSÃO}

Vários estudos têm investigado como as crianças desenvolvem a habilidade de compreender seus estados mentais e os de outras pessoas com as quais interagem e, ainda, como utilizam esta informação para explicar e predizer o comportamento social. A relação entre habilidade cognitiva e Teoria da Mente vem sendo bastante discutida. Para que a criança compreenda uma determinada situação, ela deve construir a imagem de pessoas relacionando o agente da ação e o objeto referente a esta ação; por meio do mecanismo de atenção compartilhada. Em seguida, a criança deve interpretar o comportamento comunicativo e o contexto. A habilidade de falsa crença emerge por volta dos quatro ou cinco anos de idade e pode ser considerada ponto fundamental para o desenvolvimento da linguagem infantil e da própria cognição social ${ }^{(4-7,10)}$.

Em contrapartida, estudos sobre a relação entre desenvolvimento cognitivo e o desempenho de linguagem necessitam de investigação devido ao impacto dessas inabilidades na inserção social e educacional das crianças com síndrome de Down $^{(1,2,11-23)}$.

Analisando os resultados do TVIP, que visou comparar o desempenho das crianças de ambos os grupos quanto à compreensão verbal,verificou-se diferença estatisticamente significante (Figura 1), sendo que o GD apresentou índice abaixo do terceiro desvio-padrão, com a mediana $(10,0)$ abaixo da idade classificada como própria para aplicação do teste (três anos). Tais resultados coincidem com os achados de literatura que apontam para repertório lexical restrito em indivíduos com síndrome de Down ${ }^{(1,11,12,14-16)}$.

Por outro lado, ascrianças do GC também mostraram resultado abaixo do primeiro desvio-padrão, com mediana da idade cronológica equivalente à faixa etária de seis anos e a mediana da idade ponderada, em cinco anos. Estes dados nos permitem considerar uma possível influência da condição sócio-econômica nos resultados, especialmente em relação à escolaridade dos pais (Figura 1). Os níveis de escolaridade paterna e materna tem sido apontados como fatores de proteção para o desenvolvimento de linguagem infantil por estarem diretamente vinculadosà qualidade da estimulação ambiental $^{(22)}$.

Quanto à análise da atribuição de falsa crença, o GC (Figura 2), verificou-se progressão de acertos conforme o aumento da faixa etária das crianças nas questões de nomeação (1 e 2) e na questão referente à compreensão da situação proposta (3), sendo que apenas uma das crianças não foi capaz de respondê-la. Nas questões de nomeação, as crianças tiveram baixa frequência de acertos no uso do vocábulo clipe. Cabe lembrar que para esta questão, foram aceitas respostas como "grampo", "grampinho", "ferrinho" e aplicações quanto ao uso, como por exemplo, "de prender papel". A primeira questão também não apresentou frequência de acertos adequada, pois o fato de, na caixa de lápis utilizada, existir a figura de um pássaro desenhada, o desenho funcionou como distrator, induzindo muitas crianças a responder "pássaro" para essa questão.

Quanto à questão 4 relacionada à falsa crença (Figura 2), observamos novamente progressão de respostas certas conforme o aumento da faixa etária, mostrando que embora a habilidade de falsa crença se inicie por volta dos quatro ou cinco anos de idade, aos seisela se torna uma característica consistente, no desenvolvimento infantil ${ }^{(4-7,10,21-23)}$.

$\mathrm{Na}$ análise dos resultados do GD, verificamos que a idade das crianças não influenciou a frequência de acertos em nenhuma das questões (Figura 3). Houve baixa frequência de respostas certas tanto na questão 1 quanto na 2 .

Na compreensão situacional (questão 3), os melhores resultados obtidos foram os dos indivíduos com maior tempo de terapia fonoaudiológica na instituição (Figura 4).

Não houve relação estatisticamente significante com o grau de retardo mental e as respostas às questões (Figura 5).

Quanto à questão referente à falsa crença (questão 5) houve associação entre o tempo de intervenção terapêutica e a frequência de respostas certas, porém é importante ressaltar que apenas as crianças com grau de retardo mental leve foram capazes de respondê-la (Figura 5). Esses resultados nos fazem ponderar sobre a influência positiva da intervenção terapêutica para o desenvolvimento da linguagem de crianças com síndrome de Down, especialmente quando o comprometimento intelectual é menos acentuado ${ }^{(24)}$.

Ao analisarmos a frequência de respostas certas versus erradas nas questões de falsa crença no GD, comparando-as com os resultados do TVIP (Figura 1), não verificamos correlação entre o nível de vocabulário receptivo e a habilidade de falsa crença.

Observamos diferenças estatisticamente significantes para todas as questões, entre os grupos Down e Controle (Figuras 2 e 3), com desempenho sempre melhor das crianças consideradas típicas. É importante ressaltar que tanto as dificuldades de respostas às questões de nomeação, quanto de compreensão do contexto e dafalsa crença das crianças com síndrome de Down, deveu-se muito provavelmente ao rebaixamento cognitivo ${ }^{(1,2)}$ e não a possíveis prejuízos sociais.

Finalizando, acreditamos ser importante a continuidade do estudo, considerando-se níveis sócio-econômico-cultural em diferentes em amostras de crianças típicas e de forma complementar, dar prosseguimento a estudos comparativos entre crianças com síndrome de Down, e com outros transtornos de linguagem, a fim de se caracterizar ainda mais a relação entre o desenvolvimento de linguagem e a habilidade de atribuição de falsa crença. 


\section{CONCLUSÃO}

Concluímos que a habilidade de atribuir falsa crença depende de diversos fatores, para emergir nas crianças comsíndrome de Down.

Um fator importante para a aquisição de falsa crença foi o grau de retardo mental. Apenas as crianças com retardo mental leve foram capazes de utilizá-la. Além disso, o vocabulário receptivo foi mais restrito em crianças cujo retardo mental era mais acentuado.

O tempo de terapia fonoaudiológica, na instituição, foi relevante não só para a atribuição de falsa crença, mas principalmente para a compreensão da situação proposta.

Sendo assim, foi possível avaliar a atribuição de falsa crença em crianças com síndrome de Down.

\begin{abstract}
Purpose: To evaluate the attribution of false belief in individuals with Down syndrome. Methods: Eleven children of both genders with Down syndrome and ages between four and eight years composed the Down Group (DG). All subjects used verbal communication, had mild to severe mental retardation, and were attended at the same institution. In addition, 85 children within normal development with ages between four and six years were recruited at an elementary school, constituting the Control Group (CG). The Peabody Picture Vocabulary Test (PPVT) was used to compare the level of verbal comprehension of the groups, and the adaptation of the "smarties test" was used to assess the attribution of false belief. Results: The analysis of the PPVT showed a statistically significant difference between the groups, with the DG presenting scores below the third standard deviation, and the CG, below the first standard deviation. Regarding the false belief analysis, the CG presented increasing scores in all questions as the age group increased. The same was not observed for the DG, whose subjects with best results were those that attended speech-language therapy for a longer time at the institution. No correlation was found between receptive vocabulary level and the ability of false belief. Conclusion: The CG showed a better performance in all questions of the false belief test, when compared to the DG. Thus, it was possible to evaluate false belief in children with Down syndrome.
\end{abstract}

Keywords: Down syndrome; Child language; Child development; Mental processes; Cognition

\section{REFERENCIAS}

1. Rondal JA. Síndrome de Down. In: Bishop D, Mogford K. Desenvolvimento da linguagem em circunstâncias excepcionais. Rio de Janeiro: Revinter; 2002. p. 225-42.

2. Vicari S. Motor development and neuropsychological patterns in persons with Down syndrome. Behav Genet. 2006;36(3):355-64.

3. Baldini SM, Assumpção FB. Teoria da mente - comparação entre autistas e deficientes mentais. Sinopse de Pediatria. 1999;1(1):5-16.

4. Baron-Cohen S, Leslie AM, Frith U. Does the autistic child have a "theory of mind"? Cognition. 1985;21(1):37-46.

5. De Villiers J. The Interface of Language and Theory of Mind. Lingua. 2007;117(11):1858-78.

6. Roazzi A, Santana SM. Teoria da mente: efeito da idade, do sexo e do uso de atores animados e inanimados na inferência de estados mentais. Psicol Reflex Crit. 1999;12(2): 307-30.

7. Jou GI, Sperb TM. Teoria da mente: diferentes abordagens. Psicol Reflex Crit. 1999;12(2):287-306.

8. Organização Mundial da Saúde. CID-10:classificação estatística internacional de doenças e problemas relacionados à saúde. 10a. rev. 3a ed. São Paulo: EDUSP; 1996. vol.1.

9. Capovilla FC, Capovilla AGS. Desenvolvimento linguístico na criança dos dois a seis anos: Tradução e estandardização do Peabody Picture Vocabulary Test de Dunn \& Dunn, e da Language Development Survey de Rescorla. Ciência cognitiva: Teoria Pesquisa e Aplicação. 1997;1(1):353-80.

10. Perner J, Leekman SR, Wimmer H. Three-year-olds' difficulty with false belief: the case for a conceptual deficit. Br J Dev Psychol. 1987;5(1):125-37.

11. Shwartzman JS. Aspectos da linguagem na criança com síndrome de Down. In: Shwartzman JS. Síndrome de Down. São Paulo: Mackenzie: Memnon; 1999. p. 206- 31.
12. Tristão RM, Feitosa MAG. Linguagem na Síndrome de Down. Psicol Teor Pesqui. 1998;14(2):127-37.

13. Porto E, Pereira T, Magal SAC. Análise da produção articulatória e dos processos fonológicos realizados por crianças portadoras de Síndrome de Down. Pró-Fono. 2000;12(1):34-9.

14. Bishop D, Mogford K. Desenvolvimento da linguagem em condições normais. In: Bishop D, Mogford K. Desenvolvimento da linguagem em circunstâncias excepcionais. Rio de Janeiro: Revinter; 2002. p.01-26.

15. Mancini MC, Silva PC, Gonçalves SC, Martins SM. Comparação do desempenho funcional de crianças portadoras de síndrome de Down e crianças com desenvolvimento normal aos 2 e 5 anos de idade. Arq Neuropsiquiatr. 2003;61(2B):409-15.

16. Monteiro MIB. A dinâmica do diálogo de crianças portadoras de Síndrome de Down [tese]. São Paulo: Instituto de Psicologia da Universidade de São Paulo; 1992.

17. Limongi SCO, Andrade RV, Lima FAGF, Alabarse VM, Perez VM. Processo terapêutico fonoaudiológico realizado com um par de gêmeos portadores de Síndrome de Down. Pró-Fono. 2000;12(1):24-33.

18. Andrade RV, Limongi SCO. O processo terapêutico fonoaudiológico de crianças pequenas portadoras de síndromes e orientação a família. Rev Soc Bras Fonoaudiol. 2001;6(6):29-33

19. Cardoso MCAF. Estudo de caso: atendimento fonoaudiológico de um adolescente portador de Síndrome de Down. Pró-Fono. 2001;13(2):199203.

20. Machado MLG, Montoro MF. Avaliação conjunta fonoaudiológica e psicológica em bebês portadores da síndrome de Down. Pediatr Mod. 2002;38(8):402-5

21. Dias MGBB, Soares GB, Sá TP. Conhecimento sobre a mente e compreensão sobre as intenções do experimentador. Psicol Teor Pesqui. $1994 ; 10(2): 221-9$ 
22. Santana SM, Roazzi A. Cognição social em crianças: descobrindo a influência de crenças falsas e emoções no comportamento humano. Psicol Reflex Crit. 2006;19(1):1-8.

23. Baron-Cohen S. The autistic child's theory of mind: a case of specific developmental delay. J Child Psychol Psychiatry. 1989;30(2):285-97.
24. Abbeduto L, Pavetto M, Kesin E, Weissman MD, Karadottir S, O’Brien A, Cawthon S. The linguistic and cognitive profile of Down syndrome: evidence from a comparison with fragile X syndrome. Downs Syndr Res Pract. 2001;7(1):9-15. 